\title{
Rol del pequén (Athene cunicularia) como controlador biológico mediante el análisis de sus hábitos alimentarios en la Provincia de Iquique, norte de Chile
}

\author{
Role of the burrowing owl (Athene cunicularia) as a biological \\ controller using the analysis of its food habits in the \\ Iquique province, northern Chile
}

Felipe S. Carevic ${ }^{1}$

\begin{abstract}
RESUMEN
En el presente estudio se analiza la dieta del pequén (Athene cunicularia) en la Reserva Nacional Pampa del Tamarugal y Los Verdes, provincia de Iquique, áreas que se caracterizan por las actividades campesinas y pesqueras, respectivamente. Mediante el análisis de 128 egagrópilas se han determinado un total de 422 presas. También se estudia el grado de selección de sus presas por abundancia en terreno a partir de la comparación de las abundancias de éstas en el terreno y en la dieta. La dieta del pequén estuvo compuesta casi en su totalidad por artrópodos, siendo los insectos las presas más abundantes en ambas dietas, seguida de arácnidos y mamíferos. Fueron también los artrópodos la clase de presas más abundante en terreno y el análisis dietario refleja una diferencia significativa entre las abundancias de presas en terreno versus la frecuencia de individuos en las dietas, lo cual sería un indicativo de la selectividad de presas por abundancia. A. cunicularia consume un amplio rango de especies presa, incluyendo invertebrados, arácnidos, reptiles, aves y mamíferos. Estos resultados son congruentes con estudios dietarios realizados en otras latitudes y avala la importancia de esta ave como controlador biológico de especies consideradas poco beneficiosas para los bosques de Tamarugos y sectores con alta intervención antrópica.
\end{abstract}

Palabras clave: Athene cunicularia, Dieta, Iquique, Norte de Chile, Pequén.

\begin{abstract}
In the present study the diet of burrowing owl (Athene cunicularia) was analyzed in the Pampa del Tamarugal National Reserve and Los Verdes, in the Province of Iquique. These sites are characterized by rural and fishing activities, respectively. Through the analyses of 128 pellets of the burrowing owl, 422 total prey items were determined at the two sites. An analysis of prey selectivity was performed by comparing the abundance of prey in the field and the diet of the burrowing owl. The diet of the burrowing owl at both sites included mainly arthropods. Insects were the most important prey, followed by arachnids and small mammals. The comparison between the abundance of the arthropods in the field versus the pellets of the burrowing owl indicated statistical differences, showing a degree of prey selectivity. Athene cunicularia predated on a wide range of prey, including invertebrates, reptiles, birds and small mammals. Within the invertebrates, the burrowing owl predated mainly insects. These results are congruent with studies carried out at other latitudes and confirm the burrowing owl as a biological controller of deleterious species for the Tamarugo forest and zones with anthropic activities.
\end{abstract}

Key words: Athene cunicularia, Burrowing owl, Diet, Iquique, Northern Chile.

\section{Introducción}

El pequén (Athene cunicularia) es un estrigiforme cuya distribución abarca un gran número de ambientes tanto naturales como urbanos, extendiéndose desde Canadá hasta el Sur de Chile (Meyer de Schauensee, 1970). Esta rapaz posee características de nidificación muy particulares, ya que es capaz de utilizar cuevas a nivel del suelo, que generalmente están protegidas por arbustos y restos vegetales (Schlatter et al., 1982).

Diversos estudios relacionados con la dieta del pequén han demostrado que sus hábitos alimenticios poseen un carácter generalista, consumiendo un

1 Universidad Arturo Prat, Departamento de Ciencias del Mar, casilla 121, Iquique, Chile.

E-mail: felipe.carevic@dcaf.uhu.es 
amplio rango de especies presa que abarcan tanto invertebrados como vertebrados, siendo los insectos el ítem de mayor preferencia, seguido por roedores (Meserve et al., 1987; Schlatter et al., 1982; Silva et al., 1995). Asimismo, similares características se han presentado en estudios tróficos de esta especie en áreas montañosas de los Estados Unidos (Gleason \& Johnson, 1985), bosques de Oregon (Green et al., 1993) y zonas áridas de Argentina (Nabte, 2003). Aunque en Chile los hábitos dietarios del pequén han sido bien documentados en las zonas centrales (Jaksic \& Yañez, 1978; Torres-Contreras et al., 1994) y mediterránea de Chile central (Jaksic et al., 1977; Jaksic et al., 1981), no existen datos en ambientes áridos del norte de Chile tales como el desierto de Atacama. De esta manera, el presente estudio tiene como objetivo proporcionar información sobre la dieta de esta rapaz y su rol como potencial controlador biológico en dos zonas con significación agrícola y pesquera como la pampa del Tamarugal y playa Los Verdes.

\section{Material y Métodos}

El estudio fue realizado en dos sitios del extremo norte de Chile (provincia de Iquique), ubicados en el desierto de Atacama. El primero corresponde a la Reserva Nacional Pampa del Tamarugal (RNPT) $\left(20^{\circ} 24^{\prime} \mathrm{S}, 69^{\circ} 44^{\prime} \mathrm{W}\right)$ caracterizada por un clima desértico interior con escasas precipitaciones anuales y temperaturas promedio que fluctúan entre mínimas de $5,3{ }^{\circ} \mathrm{C}$ en julio-agosto y máximas de $32,17^{\circ} \mathrm{C}$ entre los meses octubre-febrero (Lanino, 2004). La vegetación existente se restringe casi exclusivamente a bosques de tamarugos Prosopis tamarugo y algarrobos Prosopis chilensis (Gajardo, 1994; Porter, 1994). Uno de los grandes problemas para estas especies es la presencia de lepidópteros parásitos que actúan en forma nociva estableciendo sus huevos sobre el ápice de crecimiento de ramillas o el raquis de la inflorescencia, lo que conlleva a una serie de daños que afectan directamente la producción de flores y frutos (Vargas et al., 1986). Las actividades que principalmente se realizan en esta zona son crianza de ganado, la cual es llevada a cabo mayoritariamente por el campesinado de origen aymara (Carevic, 2000). El segundo sitio de muestreo se ubicó en playa Los Verdes (20 49' $\mathrm{S}$, $70^{\circ} 09^{\prime} \mathrm{W}$ ), el cual posee un clima típico de desierto costero, cuyas precipitaciones anuales no sobrepasan los 0,6 mm (Lanino, 2004), la vegetación existente en el sitio de estudio es escasa, ya que se trata de un área con abundantes desechos sólidos producto de la actividad pesquera presente en la zona.

El estudio de la alimentación se realizó mediante análisis de egagrópilas. Estas fueron colectadas dentro o en los alrededores de madrigueras activas del pequén cada dos meses entre enero y noviembre de 2004. En total se utilizaron 64 regurgitados enteros en cada uno de los dos sitios estudiados, para posteriormente, en laboratorio, separar los restos tegumentarios, dentarios, óseos y quitinosos. Cada especie presa fue determinada al mayor nivel taxonómico posible, mediante la utilización de claves especializadas (Peña, 1992; Zapfe, 1959; Reise, 1973). Aves y reptiles fueron clasificados utilizando la colección de referencia del Museo Ciencias del Mar de la Universidad Arturo Prat.

La abundancia de pequeños mamíferos fue estimada a través de cuatro transectos consistentes en tres estaciones de muestreo espaciadas cada una por intervalos de 25 metros, cada estación estuvo compuesta de cinco trampas tipo Sherman, utilizando como cebo una mezcla de avena humedecida y mantequilla de maní. Las trampas fueron colocadas durante tres noches consecutivas, siendo retiradas cada mañana para su verificación. De esta manera, el esfuerzo de trampeo empleado fue de 60 trampas/noche en cada sitio de estudio. Cada individuo capturado fue marcado y pesado mediante una pesa calibrada Pesola ${ }^{\circledR}$ 300. Los datos de abundancia resultantes fueron agrupados contabilizando el número de individuos capturados en 180 trampas.

Para la estimación de la abundancia relativa de invertebrados en cada sitio de estudio se utilizaron diez trampas de caída tipo Barber durante los meses de febrero y julio. Cada una contenía una solución de glicerina y formalina al 10\% (Topping \& Sunderland, 1992). Las trampas fueron ubicadas al azar en un área de 50 x $30 \mathrm{~m}$. Una vez retirada cada trampa se procedió a identificar cada individuo a nivel de familia, cuado fue posible.

Para la determinación de similitudes dietarias se comparó ambos sitios de muestreo, tomando en consideración la abundancia de las presas consumidas a través del índice de similitud de Morisita-Horn el cual es empleado para análisis cuantitativo de muestras (Magurran, 1988). Para la determinación de selectividad entre las variables de frecuencia de presas consumidas por el pequén y la abundancia de estas presas en terreno, se realizó una prueba 
chi-cuadrado con los datos obtenidos en cada uno de los dos sitios.

\section{Resultados}

En la RNPT se obtuvieron un total de 209 presas con una media de $3,26 \pm 1,8$ individuos por egagrópila, siendo 2 la mayor frecuencia de individuos hallados por regurgitado $(23,43 \%)$ con un rango 1-10. La composición de la dieta en este sitio reflejó que el ítem más consumido fueron los invertebrados (Tabla 1), con un $85,17 \%$. Aunque también se obtuvieron presas pertenecientes a mamíferos, reptiles y finalmente aves. Sólo dos especies de pequeños mamíferos fueron capturados durante los muestreos; el lauchón orejudo Phyllothis darwini y la rata noruega Rattus norvegicus. P. darwini presentó una mayor abundancia $(77,8 \% ; n=7)$ en tanto que solo dos individuos fueron capturados para $R$. norvegicus $(22,2 \%)$, mientras que las medias de los pesos de ambas especies correspondieron a $51,28 \pm 4,68 \mathrm{~g}$ y $168,75 \pm 37,5 \mathrm{~g}$, respectivamente. En la Tabla 2 se indica la abundancia de los artrópodos colectados mediante las trampas de caída, en la que 113 individuos fueron capturados pertenecientes a siete órdenes, siendo Coleoptera el que presentó una mayor abundancia $(42,48 \%)$ y Mantodea $(0,89 \%)$ el de menor representación.

El contenido de las egagrópilas de playa Los Verdes arrojó un total de 213 presas, con una media de 3,32 $\pm 1,9$ individuos por regurgitado, mientras que la frecuencia de presas más común hallada por egagrópila fue de $1(18,93 \%)$ con un rango de 1-9. Coleoptera $(62,91 \%)$ fue, al igual que en RNPT, el orden de presas más consumidas. En cuanto a vertebrados, fue el ratón doméstico Mus musculus el que conformó secundariamente la dieta, seguido de Scorpionida. Finalmente reptiles y aves constituyeron el total de las presas analizadas. Durante el estudio en este sitio 19 individuos de pequeños mamíferos fueron capturados, de acuerdo a estos resultados se identificó a los roedores Mus musculus $(89,47 \% ; \mathrm{n}=17)$ y Rattus norvegicus $(10,53 \% ; \mathrm{n}=2)$ como las únicas especies presentes,

Tabla 1. Composición y abundancia (F) de la dieta del pequén en dos hábitats del norte de Chile de acuerdo al análisis de 128 egagrópilas

\begin{tabular}{|c|c|c|c|c|}
\hline $\begin{array}{l}\text { Especies presa } \\
\qquad(\mathrm{n}=128)\end{array}$ & $\begin{array}{c}\text { Los Verdes } \\
\left(\mathrm{N}^{\circ} \text { individuos }\right)\end{array}$ & $\mathrm{F} \%$ & $\begin{array}{c}\text { RNPT } \\
\left(\mathrm{N}^{\circ} \text { individuos }\right)\end{array}$ & $\mathrm{F} \%$ \\
\hline \multicolumn{5}{|l|}{ MAMMALIA } \\
\hline \multicolumn{5}{|l|}{ Rodentia } \\
\hline Mus musculus & 36 & 16,9 & & \\
\hline Phyllotis darwini & & & 11 & 5,26 \\
\hline \multicolumn{5}{|l|}{ Marsupialia } \\
\hline Thylamys elegans & & & 7 & 3,35 \\
\hline \multicolumn{5}{|l|}{ REPTILIA } \\
\hline Sauria indet. & 17 & 7,98 & & \\
\hline Phyllodactylus gerrhopygus & & & 10 & 4,78 \\
\hline \multicolumn{5}{|l|}{ INSECTA } \\
\hline \multicolumn{5}{|l|}{ Coleoptera } \\
\hline Buprestidae & 45 & 21,13 & 33 & 15,79 \\
\hline Tenebrionidae & 89 & 41,78 & 62 & 29,67 \\
\hline Anobiidae & & & 4 & 1,91 \\
\hline \multicolumn{5}{|l|}{ ARACHNIDA } \\
\hline \multicolumn{5}{|l|}{ Scorpionida } \\
\hline Bothriuridae & 25 & 11,74 & 79 & 37,8 \\
\hline \multicolumn{5}{|l|}{ AVES } \\
\hline Passeriformes & 1 & 0,47 & 3 & 1,44 \\
\hline Total & 213 & 100,00 & 209 & 100,00 \\
\hline
\end{tabular}


Tabla 2. Número y abundancia relativa (F) de artrópodos hallados en los muestreos en terreno

\begin{tabular}{|c|c|c|c|c|}
\hline Orden & Los Verdes & $\mathrm{F} \%$ & RNPT & F \% \\
\hline \multicolumn{5}{|l|}{ ARACHNIDA } \\
\hline Bothriuridae & 7 & 9,72 & 13 & 11,50 \\
\hline Araneae & & & 9 & 7.97 \\
\hline \multicolumn{5}{|c|}{ HIMENOPTERA } \\
\hline Formicidae & & & 14 & 12,39 \\
\hline Apidae & & & 4 & 3,54 \\
\hline \multicolumn{5}{|l|}{ COLEOPTERA } \\
\hline Tenebrionidae & 19 & 26,39 & 21 & 18,58 \\
\hline Buprestidae & 23 & 31,94 & 11 & 9,74 \\
\hline Scarabaeidae & & & 16 & 14,16 \\
\hline \multicolumn{5}{|l|}{ ACARI } \\
\hline Indeterminado & 2 & 2,78 & & \\
\hline Tetranychidae & & & 3 & 2,65 \\
\hline \multicolumn{5}{|l|}{ DIPTERA } \\
\hline Muscidae & 14 & 19,45 & & \\
\hline Agromyzidae & & & 5 & 4,42 \\
\hline \multicolumn{5}{|l|}{ MANTODEA } \\
\hline Mantidae & & & 1 & 0,89 \\
\hline \multicolumn{5}{|l|}{ LEPIDOPTERA } \\
\hline Geometridae & 7 & 9,72 & 16 & 14,16 \\
\hline Total & 72 & 100,00 & 113 & 100,00 \\
\hline
\end{tabular}

constituyendo el peso medio de M. musculus un valor de $42,14 \pm 6,51 \mathrm{~g}$, el cual genera un notable contraste con los 200,0 $\pm 14,14 \mathrm{~g}$ de $R$. norvegicus. En cuanto a la abundancia en terreno de invertebrados, 72 artrópodos pertenecientes a cinco órdenes fueron determinados mediante las trampas Barber, al igual que en la RNPT Coleoptera fue el orden más abundante $(58,33 \%)$, y Acari $(2,78 \%)$ el menos representado.

El valor obtenido a través del índice MorisitaHorn reflejó una alta similitud $(0,857)$ entre los datos obtenidos en ambas zonas geográficas, mientras que el valor de la prueba chi cuadrado entre las distribuciones de las presas consumidas por el pequén versus las presentes en terreno en cada sitio demostraron diferencias significativas (RNPT: $\chi^{2}=527,12, \mathrm{df}=11, \mathrm{P}<0,001$; Los Verdes: $\chi^{2}=348,22$, df $\left.=5, \mathrm{P}<0,001\right)$.

\section{Discusión}

A pesar de que $A$. cunicularia consume un amplio rango de especies presa, incluyendo invertebrados, arácnidos, reptiles, aves y mamíferos, aunque se puede observar que los insectos fueron las presas más frecuentes en los regurgitados analizados en ambos sitios de estudio, la dieta del pequén es básicamente insectívora/omnívora. Esto cobra relevancia al analizar la coexistencia ecológica de otra rapaz que habita estas zonas con hábitos alimentarios totalmente diferentes como Tyto alba cuya dieta se basa prácticamente en micromamíferos (Carmona \& Rivadeneira, 2006).

Los resultados obtenidos de acuerdo al análisis estadístico nos reflejan diferencias significativas en la prueba de chi cuadrado, lo cual permite sustentar la premisa que el pequén realiza cierta selección de sus presas de acuerdo a la abundancia de sus presas en terreno. Justamente el orden Coleoptera resultó ser la presa de artrópodos más abundante en ambas áreas. Resultados similares han sido documentados para A. cunicularia en la zona semiárida de Chile los cuales asocian la abundancia de los roedores en la dieta con la presencia de éstos en terreno, en donde los roedores más abundantes tales como $P$. darwini, A. olivaceus y $O$. longicaudatus fueron los 
más consumidos dentro del análisis dietario (TorresContreras et al., 1994), también, en el presente estudio, se denota cierta selectividad del pequén en épocas como primavera donde la abundancia de pequeños mamíferos es elevada, es decir, cuando se registra un aumento de producción primaria, lo que provee abundante alimento a hembras preñadas y juveniles, es en estos meses cuando los roedores se han visto mejor representados en las egagrópilas (Figura 1), por el contrario, se documenta que la compensación a las bajas poblacionales de pequeños mamíferos en terreno se ven equilibradas con un mayor consumo de artrópodos en las dietas de pequén (Pefaur et al., 1979; Schlatter et al., 1982). A través del índice de Morisita-Horn de ambas dietas se estableció una fuerte similitud entre el número de individuos consumidos en ambos hábitats lo que nos indicaría, siguiendo el criterio de Sánchez y López (1988), que establece la similaridad de dos nichos tróficos en un valor de $66,6 \%$, que las dietas de ambos hábitats son estadísticamente símiles, hecho que se refleja a modo de ejemplo en las clases de artrópodos consumidos ya que hubo similitud tanto en la zona costera como en el área protegida en cuanto a especies consumidas.

Como se pudo apreciar, numéricamente los roedores más abundantes en ambos muestreos fueron los de mayor consumo por parte del pequén, no es coincidencia además que éstos posean un peso corporal menor al resto de las especies, ejemplo de ello es $P$. darwini por sobre $R$. norvegicus, puesto que esta rapaz tendería a aceptar presas de roedores dentro de un rango de peso de 26,3 y 85 g (Péfaur et al., 1977),

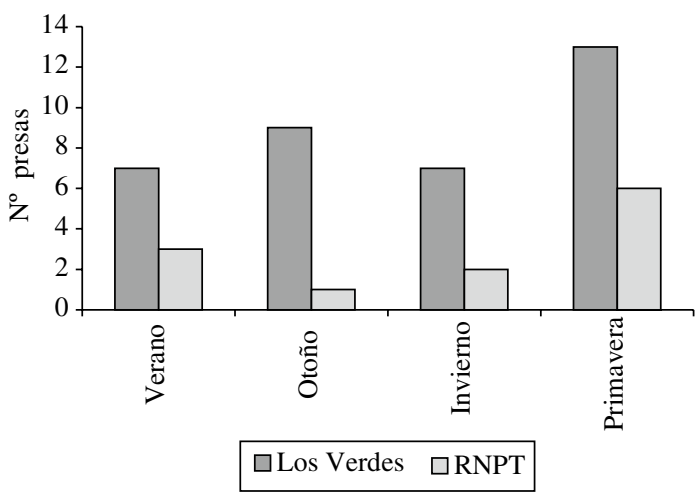

Figura 1. Distribución estacional de mamíferos presentes en ambos sitios de estudio. Resultados dados en número de individuos ( $\mathrm{N}^{\mathrm{o}}$ presas) en 128 egagrópilas. esto probablemente estaría ligado a los requerimientos energéticos de cada rapaz y a la capacidad cierta de traslado de presas de mayor tamaño puesto que la conducta de caza de los estrigiformes contemplan el hecho de llevar su presa una vez capturada a su cueva (Phelan, 1977), lo que en el caso de rapaces pequeñas como $A$. cunicularia impediría realizar ya que poseerían una superficie alar inadecuada para sustentar el peso de una presa que excediera el tamaño crítico, esta explicación cobra validez al analizar la correlación positiva dada entre el tamaño corporal y el tamaño del ala de estrigiformes chilenas estudiadas (Jaksic et al., 1977).

Este estudio constituye el primer registro de los hábitos alimentarios de $A$. cunicularia para el desierto de Atacama, el que además de posibilitarnos el análisis de su dieta, nos permite determinar las abundancias poblacionales de las especies presa que habitan el área de nidificación del pequén, variable fundamental para comprender el comportamiento trófico de esta ave. Asimismo permite clasificar a esta rapaz como un potencial controlador biológico de especies, ya que conociendo los hábitos de vida de roedores plaga como $M$. musculus o $P$. darwini, considerados como especies dañinas para tamarugos (Rodríguez, 1984), o de artrópodos que parasitan con sus huevos los frutos de tamarugos (Bobadilla et al., 1993), que están presentes en la dieta de $A$. cunicularia se incrementa aún más la necesidad de hacer estudios y formular planes de conservación de rapaces en ecosistemas frágiles como el desierto de Atacama.

\section{Conclusiones}

Los hábitos alimentarios de $A$. cunicularia analizados sobre la base de 128 egagrópilas colectadas en dos hábitats de la Provincia de Iquique mostraron una dominancia numérica de artrópodos, mamíferos y aves. Estas características denotan el hábito generalista del pequén en esta zona ya que depreda sobre un amplio ítem de presas. La mayor frecuencia de artrópodos y roedores en las dietas muestran una dependencia positiva en relación a la abundancia de micromamíferos y artrópodos en terreno, es decir, el pequén selecciona sus presas de acuerdo a la mayor abundancia de éstos en sus nichos tróficos. Selecciona además a los roedores más pequeños presentes en estas áreas, lo cual guarda 
directa relación con los requerimientos energéticos y esfuerzo de captura del roedor. Dentro de las especies de mamíferos identificadas, se describen como dañinas para especies como tamarugos y algarrobos presentes en la zona de estudio, esto sugiere la necesidad de estudiar y analizar las dietas de rapaces chilenas y compararlas con la abundancia de las especies presa en el hábitat de residencia del ave, lo cual clarificaría aún más la ecología trófica y la capacidad cierta de esta ave como biocontrolador en sistemas agrícolas.
Agradecimientos. A la Universidad Arturo Prat, en especial al departamento de agricultura del desierto y biotecnología por el soporte dado al autor en la adquisición del material de muestreo; a Conaf por la facilitación de sus instalaciones en la Reserva Nacional Pampa del Tamarugal. A Walter Sielfeld por su valiosa ayuda en las tareas de terreno y elaboración del artículo. A Erico Carmona, Miguel Valdebenito y César Cardozo por la ayuda prestada durante la fase de muestreo de la presente investigación.

\section{Literatura Citada}

Bobadilla, D.; Vargas H.; Jiménez, H.

1993 Enemigos naturales de los principales lepidópteros plagas del tamarugo. Idesia. 12: 6-9.

Carevic, A.

2000 Los campesinos del desierto chileno. Ediciones Campus. Universidad Arturo Prat. Iquique, Chile. 222 p.

Carmona, E.; Rivadeneira, M.

2006 Foods habits of the barn owl Tyto alba in National Reserve Pampa del Tamarugal, Atacama desert, North Chile. J. Nat. Hist. 40: 473-483.

Gajardo, R.

1994 La vegetación natural de Chile. Clasificación y distribución geográfica. Colección Imagen de Chile. Editorial Universitaria. Santiago, Chile. 165 p.

Gleason, R.; Johnson, D.

1985 Factors influencing nesting success of burrowing owls in southeastern Idaho. Great Basin Nat. 45 (1): 81-84.

Green, G.; Fitzner, R.; Anthony, R.; Rogers, L.

1993 Comparative diets of burrowing owls in Oregon and Washington. Northwest Sci. 67 (2): 88-93.

Jaksic, F.; Yáñez, J.; Persico, R.; Torres, J.

1977 Sobre la partición de recursos por las Strigiformes de Chile central. Anales del Museo de Historia Natural de Valparaíso. 10: 185-194.

Jaksic, F.; Yáñez, J.

1978 Predación oportunista de Speotyto cunicularia en el norte chico. Noticiario Mensual del Museo Nacional de Historia Natural. 272: 12.

Jaksic, F.; Greene, H.; Yáñez, J.

1981 The guild structure of a community of predatory vertebrates in central Chile. Oecologia. 49: 21-28.

Lanino, $\mathrm{M}$.

2004 Antecedentes climáticos de la Estación Experimental Canchones, en la Pampa del Tamarugal. Revista Agricultura del Desierto. Universidad Arturo Prat. Iquique, Chile. 3: 57-68.

Magurran, A.

1988 Ecological diversity and its measurement. Princeton University Press, New Jersey, USA, 179 p.

Meserve, P.; Shadrick, E.; Kelt, D.

1987 Diet and selectivity of two chilean predators in the northern semi-arid zone. Rev. Chil. Hist. Nat. 60: 93-99.
Meyer de Schaunsee, R.

1970 A guide to the birds of South America. The Academy of Natural Sciences of Philadelphia. 470 p.

Nabte, M.

2003 Dieta de Athene cunicularia (Aves: Strigiformes) en el nordeste de la provincia del Chubut, Argentina. Resúmenes de tesis. Mastozoología Neotropical. 11: 249-254.

Pefaur, J.; Jaksic, F.; Yáñez, J.

1977 La alimentación de Speotyto cunicularia (Aves: Strigiformes) en la provincia de Coquimbo. Anales Museo de Historia Natural de Valparaíso. 10: 178-183.

Pefaur, J.; Yáñez, J.; Jaksic, F.

1979 Biological and environmental aspects of a mouse outbreak in the semi-arid region of Chile. Mammalia. 43: 313-322.

Peña, $L$.

1992 Introducción a los insectos de Chile. Editorial Universitaria. Tercera Edición. Santiago, Chile. 255 p.

Phelan, F.

1977 Food caching in the screech owl. Cóndor. 79: 127.

Porter, C.

1994 Perspectiva del control biológico en la Pampa del Tamarugal: aspectos biogeográficos y paleoecológicos. Idesia. 13: 81-90.

Reise, D.

1973 Clave para la determinación de los cráneos de marsupiales y roedores chilenos. Gayana. 27: 1-20.

Rodríguez, J.

1984 Roedores plagas, un peligro potencial para el tamarugo (Prosopis tamarugo). En: Estado actual del conocimiento sobre Prosopis tamarugo. Editado por Mario Habit, Oficina Regional de FAO para América Latina y el Caribe. 419421.

Sánchez, O.; López, G.

1988 A theoretical analysis of some indices of similarity as applied to biogeography. Folia Entomol. Mexic. 75: 119145

Schlatter, R.; Yáñez, J.; Nuñez, H.; Jaksic, F.

1982 Estudio estacional de la dieta del Pequén, Athene cunicularia (Molina) (Aves, Strigidae) en la Precordillera de Santiago. Medio Ambiente. 6 (1): 9-18. 
Silva, S.; Lazo, I.; Silva-Aránguiz, E.; Jaksic, F.; Meserve, P.; Gutiérrez, J.

1995 Numerical and functional response of burrowing owls to long-term mammal fluctuations in Chile. J. Raptor Res. 29: 250-255.

Topping, C.; Sunderland, K.

1992 Limitations to the use of pitfalls traps in ecological studies exemplified by a study of spiders in a field of winter wheat. J. Appl. Ecol. 29: 485-491.
Torres-Contreras, H.; Silva-Aránguiz E.; Jaksic, F. 1994 Dieta y selectividad de presas de Speotyto cunicularia en una localidad semiárida del norte de Chile a lo largo de siete años (1987-1993). Rev. Chil. Hist. Nat. 67: 329-340.

Vargas, H.; Bobadilla, D.; Aguilera, A.

1986 Adaptación morfológica y hábito de oviposición de la Polilla del botón floral del Tamarugo, Ithome sp. (Lepidóptera, Walshiidae). Idesia. 10: 39-44.

Zapfe, $\mathrm{H}$.

1959 Clave para determinar familias y géneros de arañas chilenas. Investigaciones Zoológicas Chilena. 5: 133-140. 
